\title{
Avaliação do desenvolvimento psicomotor de crianças com Síndrome de Down
}

\author{
Evaluation of psychomotor development \\ in children with Down Syndrome
}

\author{
Evaluación del desarrollo psicomotor \\ de niños con Sindrome de Down
}

\author{
Vanessa Maria Hendges ${ }^{1}$, Magali Teresinha Quevedo Grave ${ }^{2}$, Eduardo \\ Périco ${ }^{3}$
}

1.Acadêmica do curso de Fisioterapeuta. Universidade do Vale do Taquari - UNIVATES, Lajeado-RS, Brasil. 2.Fisoterapeuta, Doutora, docente da Universidade do Vale do Taquari - UNIVATES, Lajeado-RS, Brasil. 3.Biólogo, Doutor, docente da Universidade do Vale do Taquari - UNIVATES, Lajeado-RS, Brasil.

\begin{abstract}
Resumo
Introdução. A Síndrome de Down (SD) é uma alteração genética que ocorre devido à trissomia do cromossomo 21. Além do fenótipo característico, há hipotonia muscular generalizada, deficiência intelectual e atraso no desenvolvimento psicomotor (DPM). Objetivo. Verificar o DPM de crianças com SD, de até 42 meses, considerando os domínios cognitivo, de linguagem e motor. Método. Estudo exploratório, descritivo, transversal, de abordagem quantitativa, no qual participaram 13 crianças com SD, com idades entre $10 \mathrm{e}$ 40 meses, avaliadas através da Escala de Desenvolvimento Infantil Bayley III. Resultados. A pontuação escalonada demonstra que na área cognitiva, doze crianças estão a 1 DP (desvio padrão) abaixo da média e uma criança está entre 1 e 2 DP abaixo da média; na comunicação receptiva uma criança está na média, 10 crianças estão a 1 DP abaixo da média e duas crianças estão entre 1 e 2 DP abaixo da média; na comunicação expressiva uma criança está na média, cinco crianças estão a 1 DP abaixo da média e sete estão entre 1 e 2 DP abaixo da média. Na motricidade fina, uma criança está na média e 12 estão a 1 DP abaixo da média; na motricidade grossa há duas crianças a 1 DP abaixo da média, 9 crianças entre 1 e 2 DP abaixo da média e duas crianças entre 2 e 3 DP abaixo da média. Conclusão. As crianças apresentam atraso em todas as áreas do DPM, quando comparadas com crianças típicas e as áreas mais defasadas são a motricidade grossa e a comunicação expressiva.
\end{abstract}

Unitermos. Síndrome de Down; Desenvolvimento psicomotor; Escala Bayley

\footnotetext{
Abstract

Introduction. Down Syndrome (DS) is a genetic alteration that occurs due to the trisomy 21. Besides the characteristic phenotype there is widespread muscular hypotonia, intellectual disability and delay in psychomotor development (PMD). Objective. To verify PMD in children with DS up to 42 months considering cognitive, language and motor domains. Method. Exploratory study, descriptive, transversal with a qualitative approach in which participated 13 children with ages between 10 and 40 months, who were evaluated using Bayley's III Child Development Scale. Results. Phased score indicates that in the cognitive area twelve children are 1 SD (standard deviation) below the average and one child is between 1 and 2 SD below it. In the receptive communication one child is on the average, 10 are 1 SD below it and two are between 1 and 2 SD below it. In the expressive communication one child is on the average, five are 1 SD below it and seven are 1 and 2 SD below it. In the fine motor one child is on the average and 12 are 1 SD below it. In the gross motor there are two children 1 SD below the average, 9 between 1 and 2 SD below it and two between 2 and 3 SD below it. Conclusion.
} 
Children present delay in every area of PMD when compared to typical children and the most lagged areas are gross motor and expressive communication.

Keywords. Down Syndrome; Psychomotor Development; Bayley's Scale

\begin{abstract}
Resumen
Introducción. El síndrome de Down (SD) es una alteración genética que ocurre debido a la trisomía del cromosoma 21. Además del fenotipo característico, hay hipotonía muscular generalizada, discapacidad intelectual y desarrollo psicomotor retardado (MPD). Objetivo. Verificar el DPM de niños con SD, hasta 42 meses, considerando los dominios cognitivos, del lenguaje y motor. Método. estudio exploratorio, descriptivo, transversal con un enfoque cuantitativo, en el que participaron 13 niños de 10 a 40 meses, evaluados mediante la Escala de Desarrollo Infantil Bayley III. Resultados. El puntaje escalado muestra que en el área cognitiva, doce niños están 1 SD (desviación estándar) por debajo del promedio y un niño está entre 1 y 2 SD por debajo del promedio; en comunicación receptiva, un niño está en promedio, 10 niños están 1 SD por debajo del promedio y dos niños están entre 1 y 2 SD por debajo del promedio; en comunicación expresiva, un niño tiene un promedio de cinco, cinco niños están 1 SD por debajo del promedio y siete tienen entre 1 y 2 SD por debajo del promedio. En habilidades motoras finas, un niño es promedio y 12 son 1 SD por debajo del promedio; En las habilidades motoras gruesas hay dos niños con 1 DE por debajo del promedio, 9 niños entre 1 y 2 DE por debajo del promedio y dos niños entre 2 y 3 DE por debajo del promedio. Conclusión. los niños se retrasan en todas las áreas del DPM, en comparación con los niños típicos y las áreas más rezagadas son las habilidades motoras gruesas y la comunicación expresiva.
\end{abstract}

Palabras clave. Síndrome de Down; Desarrollo psicomotor; Escala de Bayley

Trabalho realizado na Universidade do Vale do Taquari - UNIVATES, Lajeado-RS, Brasil.

\title{
INTRODUÇÃO
}

A Síndrome de Down (SD), citada pelo médico britânico John Langdon Haydon Down, em 1866, é caracterizada como uma condição genética, com sinais e sintomas característicos. O fenótipo é marcado por rosto plano e largo, bochechas arredondadas, olhos oblíquos e distantes um do outro, lábios finos, língua longa e grossa, nariz pequeno, dentre outras características ${ }^{1}$. Ressalta-se que a hipotonia muscular generalizada, a deficiência intelectual e o atraso no desenvolvimento psicomotor são comuns ${ }^{2}$. Segundo as 
diretrizes de atenção à pessoa com SD, no Brasil, existe uma criança com SD para cerca de 600 a 800 nascidos vivos, independente de etnia, gênero ou classe social ${ }^{3}$.

Muitas vezes, os pais e os familiares recebem 0 diagnóstico de SD apenas na hora do nascimento, o que causa angústia, frustração e insegurança com relação ao desenvolvimento de seu filho. Nesse sentido, é importante que os profissionais estejam preparados para orientar e acolher os pais que passam por momentos difíceis ao terem um filho que foge dos padrões de normalidade impostos pela sociedade 4 .

Assim, avaliar precocemente o desenvolvimento de crianças com SD, a fim de estabelecer condutas terapêuticas que minimizem os possíveis déficits e estimulem as potencialidades, faz-se necessário, tendo em vista que estudos comprovam que a estimulação precoce e terapias como fisioterapia, fonoaudiologia, terapia ocupacional, dentre outras, costumam favorecer a aquisição de habilidades psicomotoras, principalmente nos primeiros anos de vida, que são cruciais para a plasticidade neural. A psicomotricidade também tem sido uma ferramenta importante para estimular crianças com SD; está relacionada ao DPM, contribuindo para maior autonomia e independência das crianças ${ }^{5,6}$.

Situar o desenvolvimento do bebê, considerando as áreas motora, cognitiva e de linguagem pode ser importante na definição de estratégias para estimulação do 
desenvolvimento de crianças com SD, justificando-se, assim, a realização deste estudo.

Um instrumento que vem sendo bastante utilizado pela comunidade científica na avaliação do DPM é a Escala Bayley de Desenvolvimento Infantil (Bayley III), protocolo lúdico, flexível, com excelente padrão de validade e confiabilidade, que permite a identificação precoce de problemas e/ou atraso no desenvolvimento de crianças de até 42 meses e que sinaliza a necessidade de avaliação e tratamento mais aprofundado em área(s) específica(s) ${ }^{6}$.

A Bayley III indica os pontos fortes, fracos e as competências do bebê e da criança pequena para o planejamento adequado de uma intervenção terapêutica, além do acompanhamento da evolução das intervenções, auxiliando na elaboração de um trabalho consistente de estimulação em equipe interdisciplinar?

O objetivo deste estudo foi verificar o desenvolvimento psicomotor de crianças com SD, de até 42 meses, por meio da Escala de Desenvolvimento do bebê e da criança - Bayley III, considerando as dimensões cognitiva, de linguagem (comunicação receptiva e comunicação expressiva) e de motricidade (grossa e fina), a fim de identificar a fase de desenvolvimento em que as crianças se encontram, bem como, determinar os equivalentes de idade em que as habilidades descritas acima, normalmente, são desenvolvidas, identificando-se as áreas de maior defasagem no desenvolvimento das crianças com SD participantes do estudo. 


\section{MÉTODO}

\section{Amostra}

O estudo iniciou após aprovação do Comitê de Ética e Pesquisa (COEP) da Universidade do Vale do Taquari UNIVATES, de Lajeado/RS, sob parecer de número: 3.105.228 (CAEE: 03427218.9.0000.5310). A coleta dos dados foi realizada na Clínica Escola de Fisioterapia (CEF) da referida Instituição de Ensino Superior, sendo a amostra composta por 13 crianças com SD: 6 que recebem atendimento de fisioterapia na CEF e 7 que foram convidadas a participar por meio de um grupo de WhatsApp de pais de crianças com SD, no qual a mãe de uma das crianças atendidas na CEF faz parte. As crianças participantes do estudo realizaram estimulação precoce, fisioterapia e acompanhamento fonoaudiólogo em algum momento das suas vidas.

Os participantes deveriam obedecer aos seguintes critérios de inclusão: ter diagnóstico de SD, com idades entre 1 a 42 meses; não apresentar condições associadas à SD como autismo, cardiopatias graves, dentre outras. Pais e/ou responsáveis terem assinado o Termo de Consentimento Livre e Esclarecido (TCLE).

\section{Procedimento}

Antes da avaliação de cada uma das crianças participantes, identificadas pela letra $C$, seguida de números $(1,2,3$ etc. $)$, foi realizado o cálculo da idade cronológica em meses e dias para identificação do ponto inicial das 
atividades que seriam propostas a cada criança, conforme orientações descritas no próprio instrumento padronizado da Bayley III, que possui uma escala para determinar o ponto de início e a primeira atividade a ser executada, conforme a idade cronológica da criança.

A aplicação seguiu as diretrizes do manual de administração: a sala utilizada estava livre de distrações, silenciosa, iluminada e confortável. Os materiais que compõem o kit da escala Bayley III foram dispostos de maneira que ficassem próximos da pesquisadora para facilitar o acesso no momento da avaliação.

Inicialmente, foi posicionada uma mesa pequena e duas cadeiras, nas quais ficavam sentadas frente a frente, pesquisadora e criança, de modo que a criança pudesse olhar e manusear os brinquedos disponibilizados para manuseio e exploração. Na sequência, a criança era incentivada e/ou levada até o tatame de piso emborrachado para que a pesquisadora pudesse observar o desenvolvimento da motricidade grossa e forma de uso da escada pequena de três degraus, conforme sugerido pelo manual, quando a criança demonstrasse condições motoras para tal. No momento da avaliação, permaneceram na sala a criança, o avaliador e o responsável.

As atividades foram realizadas na ordem sequencial em que estão dispostas no formulário de registro da Bayley III, ou seja: avaliação cognitiva (91 questões), escala de linguagem que se divide em comunicação receptiva (49 questões) e comunicação expressiva (48 questões), 
motricidade fina (66 questões) e, por último, avaliou-se a motricidade grossa (72 questões) ${ }^{7}$.

A aplicação do teste seguiu a regra de reversão da Bayley III que consiste em a criança, obrigatoriamente, obter pontuação igual a 1 nos 3 primeiros itens consecutivos em seu ponto inicial que são específicos para sua idade e, assim, poder prosseguir para o item seguinte, conforme disposto no protocolo. Caso a criança obtivesse 0 em alguma das 3 primeiras questões, deveria voltar ao ponto inicial para a idade anterior e realizar as atividades da idade anterior até atingir o critério de interrupção. Para a interrupção do teste, a criança deveria obter pontuação igual a 0 em 5 itens consecutivos testados. O teste consiste em pontuar 1 ou 0 nas atividades propostas, sendo essa a pontuação padrão7. O tempo de aplicação do protocolo variou de $01 \mathrm{~h}$ a 01h30min.

Por meio da Escala de Desenvolvimento Infantil - Bayley III é possível gerar-se as pontuações "bruta" "escalonada", "composta", bem como, classificação em percentis, intervalos de confiança e correlação desses resultados com gráficos e cálculos de discrepância, que podem ser utilizadas conforme a finalidade de cada estudo. Neste artigo estão apresentadas as pontuações "bruta", "escalonada", "composta", equivalência de idade de desenvolvimento e classificação descritiva, conforme explicado a seguir.

As pontuações brutas totais para a escala cognitiva, comunicação receptiva, expressiva, motricidade fina e motricidade grossa são calculadas contabilizando-se o 
número total de itens que a criança pontuou, mais o número de itens não administrados anteriormente ao nível em que ela iniciou a testagem? ${ }^{7}$.

A pontuação escalonada representa o desempenho de uma criança em relação aos seus pares da mesma idade; possui uma variação de 1 a 19 pontos, com média de 10 e desvio padrão (DP) de 3. Pontuações de 7 e 13 são equivalentes a 1 DP abaixo e acima da média, respectivamente. As pontuações 4 e 16 são equivalentes a 2 DP em relação a média?.

A pontuação composta é derivada da pontuação escalonada; é dimensionada e pode variar de 40 a 160 pontos, com média de 100 e desvio padrão de 15 pontos. Pontuações de 85 e 115 pontos equivalem a 1 DP abaixo e acima da média respectivamente; pontuações de 70 e 130 correspondem a 2 DP e, pontuações de 55 e 145 pontos correspondem a 3 DP7. Em ambas as pontuações, quanto mais alto o número de desvios padrões, mais distante da média de desenvolvimento de uma determinada faixa etária a criança se encontra.

A pontuação de equivalentes de idade de desenvolvimento e as classificações descritivas costumam ser utilizadas em conjunto com as pontuações escalonadas e compostas, a fim de retratar o desempenho da criança. Os equivalentes de idade representam a média da idade, em meses, em que uma pontuação bruta total é típica; o protocolo traz uma tabela na qual é possível verificar, conforme pontuação bruta atingida, qual a idade cronológica 
de desenvolvimento que a criança avaliada se encontra. As classificações descritivas são realizadas com base na pontuação composta e são representadas da seguinte forma: muito superior (130 e acima), superior (120-129), média elevada (110-119), média (90-109), média baixa (80-89), limítrofe (70-79) e extremamente baixa (69 e abaixo) ${ }^{7}$.

Vale ressaltar que a relação da pontuação bruta com as demais pontuações presentes na escala Bayley III estão expostas no manual de administração; basta transformar a pontuação bruta conforme correlação da tabela de equivalência, levando em consideração a idade cronológica da criança e resultados obtidos na avaliação.

\section{RESULTADOS}

Participaram do estudo 13 crianças com diagnóstico clínico de SD, sendo 7 do sexo feminino e 6 do sexo masculino, com idades cronológicas de 10 meses a 40 meses e 18 dias, na data da avaliação. A pesquisa buscou verificar o DPM de crianças com SD, tendo em vista os domínios cognitivos, de linguagem (comunicação receptiva e expressiva) e motor (motricidade grossa e fina), para identificação do funcionamento do desenvolvimento dos participantes, a fim de verificar $\mathrm{a}(\mathrm{s})$ área(s) de maior defasagem, através da Escala de Desenvolvimento infantil Bayley III.

Na Tabela 1 é possível verificar a pontuação bruta, a escalonada, a composta e o equivalente de idade, 
verificando, assim, quantos meses de atraso a criança possui em cada uma das áreas avaliadas. Na área cognitiva é possível observar que a criança mais nova possui 2 meses de atraso no desenvolvimento, enquanto a mais velha apresenta 12 meses de atraso. Na área da linguagem, tanto na comunicação expressiva, quanto na comunicação receptiva, a criança mais nova apresenta pontuação adequada para sua idade cronológica, enquanto a mais velha possui 12 meses de atraso na comunicação receptiva e 19 meses de na comunicação expressiva. Tanto na motricidade fina quanto na motricidade grossa, a criança mais nova apresentou-se com 2 meses de atraso no desenvolvimento e a mais velha com 9 meses na motricidade fina e 20 meses na motricidade grossa.

Na Figura 1 é possível verificar as crianças que estão na média de desenvolvimento, o DP em que elas se encontram. Ao observar-se a pontuação escalonada, pode-se identificar que na área cognitiva, 12 crianças estão a 1 DP abaixo da média e uma criança se encontra entre 1 e 2 DP abaixo da média; na área de linguagem em comunicação receptiva uma criança está na média, 10 crianças estão a 1 DP abaixo da média e duas crianças se encontram entre 1 e 2 DP abaixo da média; na comunicação expressiva uma criança se apresentou na média, 5 crianças estão a 1 DP abaixo da média e 7 estão entre 1 e 2 DP abaixo da média. 
Tabela 1. Pontuações bruta, equivalente de idade, escalonada e composta.

\begin{tabular}{|c|c|c|c|c|c|c|}
\hline Criança & IM & Áreas & $\begin{array}{c}\text { Pontuação } \\
\text { Bruta }\end{array}$ & $\begin{array}{c}\text { Equivalente de idade } \\
\text { (meses) }\end{array}$ & $\begin{array}{l}\text { Pontuação } \\
\text { escalonada* }\end{array}$ & $\begin{array}{l}\text { Pontuação } \\
\text { composta** }\end{array}$ \\
\hline \multirow{5}{*}{$\mathrm{C} 1$} & \multirow{5}{*}{$\begin{array}{c}10 \\
\text { meses }\end{array}$} & Cognitivo & 33 & 8 & 8 & 90 \\
\hline & & CR & 13 & 10 & 10 & \multirow{2}{*}{100} \\
\hline & & $\mathrm{CE}$ & 12 & 10 & 10 & \\
\hline & & MF & 23 & 8 & 7 & \multirow{2}{*}{82} \\
\hline & & MG & 33 & 8 & 7 & \\
\hline \multirow{5}{*}{$\mathrm{C} 2$} & \multirow{5}{*}{$\begin{array}{c}12 \\
\text { meses } \\
\text { e } 7 \\
\text { dias }\end{array}$} & Cognitivo & 37 & 10 & 8 & 90 \\
\hline & & $\mathrm{CR}$ & 12 & 9 & 7 & \\
\hline & & $\mathrm{CE}$ & 10 & 8 & 6 & 79 \\
\hline & & MF & 27 & 10 & 8 & \\
\hline & & MG & 33 & 8 & 4 & 76 \\
\hline \multirow{5}{*}{ C3 } & \multirow{5}{*}{$\begin{array}{c}13 \\
\text { meses } \\
\text { e } 9 \\
\text { dias }\end{array}$} & Cognitivo & 39 & 11 & 7 & 85 \\
\hline & & CR & 13 & 10 & 7 & \multirow[b]{2}{*}{89} \\
\hline & & $\mathrm{CE}$ & 15 & 12 & 9 & \\
\hline & & MF & 27 & 10 & 7 & \multirow{2}{*}{67} \\
\hline & & MG & 31 & 8 & 2 & \\
\hline \multirow{5}{*}{$\mathrm{C} 4$} & \multirow{5}{*}{$\begin{array}{c}21 \\
\text { meses } \\
\text { e } 15 \\
\text { dias }\end{array}$} & Cognitivo & 50 & 17 & 7 & 85 \\
\hline & & $\mathrm{CR}$ & 20 & 18 & 8 & 83 \\
\hline & & $\mathrm{CE}$ & 19 & 16 & 6 & 83 \\
\hline & & MF & 34 & 18 & 8 & \\
\hline & & MG & 46 & 14 & 5 & 19 \\
\hline \multirow{5}{*}{ C5 } & & Cognitivo & 56 & 20 & 8 & 90 \\
\hline & 22 & $\mathrm{CR}$ & 20 & 18 & 7 & \\
\hline & $\begin{array}{c}\text { meses } \\
\text { e } 15\end{array}$ & CE & 20 & 17 & 6 & 19 \\
\hline & dias & MF & 35 & 20 & 8 & 82 \\
\hline & & MG & 48 & 16 & 6 & 82 \\
\hline & & Cognitivo & 60 & 22 & 9 & 95 \\
\hline & 23 & $\mathrm{CR}$ & 23 & 20 & 9 & \\
\hline $\mathrm{C} 6$ & $\begin{array}{c}\text { meses } \\
\text { e } 4\end{array}$ & $\mathrm{CE}$ & 19 & 16 & 5 & 83 \\
\hline & dias & MF & 38 & 23 & 10 & \\
\hline & & MG & 48 & 16 & 6 & 88 \\
\hline & & Cognitivo & 59 & 22 & 8 & 90 \\
\hline & 23 & $\mathrm{CR}$ & 22 & 19 & 8 & \\
\hline $\mathrm{C7}$ & $\begin{array}{c}\text { meses } \\
\text { e } 6\end{array}$ & $\mathrm{CE}$ & 23 & 19 & 7 & 86 \\
\hline & dias & MF & 37 & 22 & 9 & \\
\hline & & MG & 46 & 14 & 4 & 19 \\
\hline & & Cognitivo & 57 & 21 & 7 & 85 \\
\hline & 24 & $\mathrm{CR}$ & 24 & 21 & 8 & \\
\hline $\mathrm{C} 8$ & meses & CE & 20 & 17 & 5 & 19 \\
\hline & dias & MF & 35 & 20 & 7 & \\
\hline & & MG & 50 & 18 & 6 & 19 \\
\hline & & Cognitivo & 60 & 22 & 8 & 90 \\
\hline & $\begin{array}{c}24 \\
\text { meses }\end{array}$ & $\mathrm{CR}$ & 23 & 20 & 8 & \\
\hline C9 & $\begin{array}{c}\text { meses } \\
\text { e } 24\end{array}$ & CE & 25 & 20 & 7 & 86 \\
\hline & dias & MF & 37 & 22 & 8 & \\
\hline & & MG & 51 & 18 & 7 & 85 \\
\hline & & Cognitivo & 61 & 23 & 9 & 95 \\
\hline & $\begin{array}{c}25 \\
\text { meses }\end{array}$ & CR & 20 & 18 & 6 & \\
\hline C10 & e 01 & CE & 24 & 19 & 7 & 19 \\
\hline & dia & MF & 38 & 23 & 9 & \\
\hline & & MG & 44 & 13 & 3 & 16 \\
\hline & & Cognitivo & 57 & 21 & 7 & 85 \\
\hline & $\begin{array}{c}25 \\
\text { meses }\end{array}$ & $\mathrm{CR}$ & 21 & 19 & 7 & \\
\hline C11 & e 20 & CE & 24 & 19 & 7 & 83 \\
\hline & dias & MF & 38 & 23 & 9 & \\
\hline & & MG & 48 & 16 & 5 & 82 \\
\hline & & Cognitivo & 59 & 22 & 6 & 80 \\
\hline & $\begin{array}{c}29 \\
\text { meses }\end{array}$ & $\mathrm{CR}$ & 21 & 19 & 6 & \\
\hline C12 & e 23 & $\mathrm{CE}$ & 24 & 19 & 6 & 17 \\
\hline & dias & MF & 42 & 28 & 9 & \\
\hline & & MG & 41 & 11 & 1 & 70 \\
\hline & & Cognitivo & 69 & 28 & 7 & 85 \\
\hline & 40 & CR & 30 & 28 & 7 & \\
\hline C13 & e 18 & CE & 27 & 21 & 5 & 17 \\
\hline & $\begin{array}{l}\mathrm{e} 18 \\
\text { dias }\end{array}$ & MF & 44 & 31 & 7 & \\
\hline & & MG & 54 & 20 & 4 & 73 \\
\hline
\end{tabular}


Figura 1. Pontuação escalonada representado pela curva de desvio padrão.

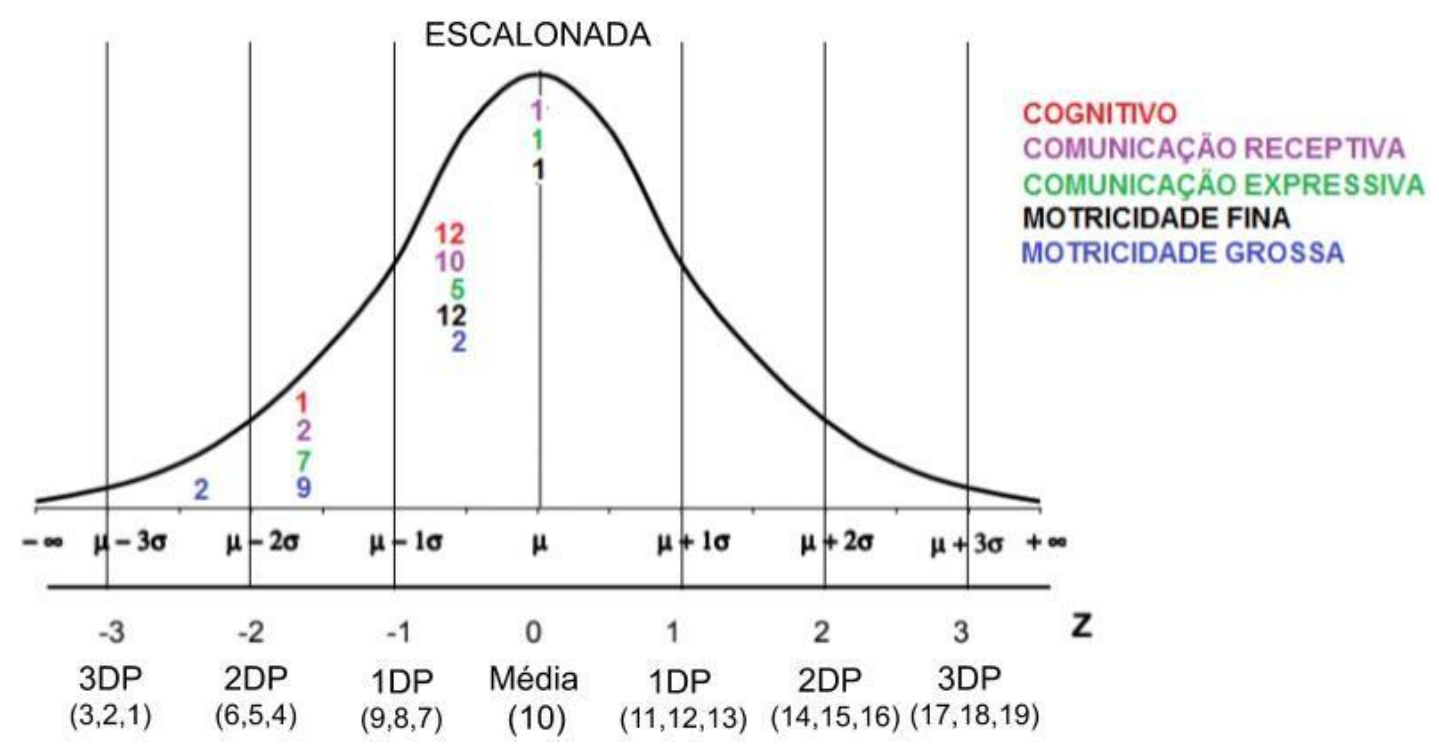

Com relação à motricidade fina observa-se que uma criança está na média e 12 estão equivalentes a 1 DP abaixo da média. Na perspectiva da motricidade grossa há duas crianças a 1 DP abaixo da média, 9 crianças entre 1 e 2 DP abaixo da média e duas crianças entre 2 e 3 DP abaixo da média.

Na Tabela 2, é possível observar a pontuação composta e a classificação descritiva de cada criança. Por meio dessa análise, verifica-se que na área cognitiva, a criança tende a se manter na média quanto menos idade ela tem e, gradativamente, a diminuir seu desempenho para média baixa conforme vai tendo mais idade.

$\mathrm{Na}$ área de linguagem, 6 crianças estão no limite do que se espera para suas faixas etárias, 6 apresentam média baixa e uma está na média. Referente à motricidade, é comum que quanto mais perto de atingir marcos motores 
antigravitários, mais defasadas as crianças se encontram, mantendo-se nos níveis de média baixa e limítrofe.

A partir dos resultados obtidos neste estudo, pode-se constatar que as áreas de maior defasagem, de modo geral, independente da idade, são: motricidade grossa e comunicação expressiva. A pontuação composta segue a mesma lógica que a pontuação escalonada.

Vale salientar que a Bayley III reconhece o uso de ajustes simples para crianças com deficiência, a fim de oferecer uma oportunidade significativa para que as habilidades da criança, que está em processo de avaliação, relacionadas ao conhecimento, sejam demonstradas e valorizadas. Assim, o julgamento do profissional deve ser usado na tomada de decisões sobre possíveis adaptações.

A Escala de Desenvolvimento Infantil Bayley III foi administrada a populações específicas, com alguns tipos de alterações psicomotoras, para que assim fosse possível haver uma comparação quando aplicada pelo avaliador nesse mesmo público.

$\mathrm{Na}$ Tabela 3, é possível verificar que o desempenho médio dos participantes do presente estudo nos domínios cognitivo, de linguagem e motor, quando comparados ao desempenho médio dos participantes do estudo realizado com as 90 crianças com SD pelos administradores da Bayley III, está mais alto e mais próximo da média do grupo controle, que era integrado por 1700 crianças com desenvolvimento típico. 
Tabela 2. Pontuação composta e classificação descritiva do desenvolvimento em cada área avaliada.

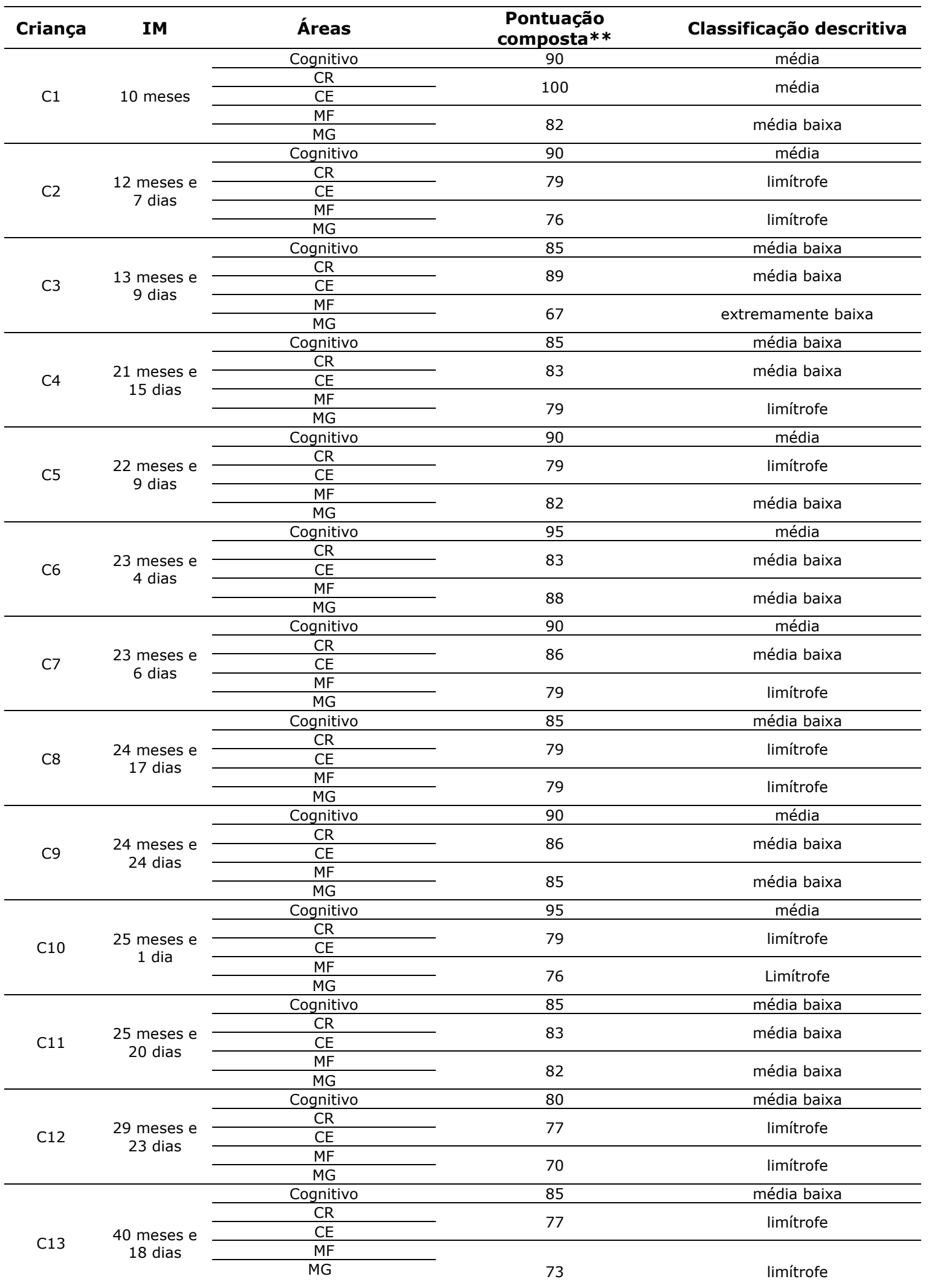

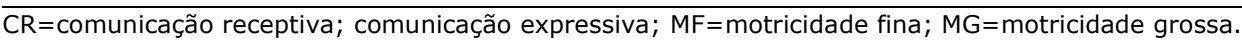


Da mesma forma, é possível observar que a média atingida pelos participantes do presente estudo fica abaixo da média de desenvolvimento do grupo controle, de crianças típicas, já esperado, pelo fato das crianças aqui avaliadas terem diagnóstico de SD. As áreas de comunicação expressiva e motricidade grossa foram identificadas como as mais defasadas.

Tabela 3. Desempenho médio dos grupos Síndrome de Down e grupo controle conforme o estudo feito pela Escala Bayley e desempenho médio verificado no presente estudo.

\begin{tabular}{cccc}
\hline Áreas/escalonada & Média da SD - Bayley & $\begin{array}{c}\text { Média de grupo } \\
\text { controle }\end{array}$ & Média SD - estudo \\
\hline Cognitivo & 3,6 & 10,6 & 7,61 \\
\hline Comunicação receptiva & 5,1 & 10,6 & 7,53 \\
\hline Comunicação expressiva & 4,9 & 10,5 & 6,61 \\
\hline Motricidade fina & 4,0 & 10,4 & 8,15 \\
\hline Motricidade grossa & 3,4 & 10,3 & 4,61 \\
\hline Áreas/composta & Média da SD - Bayley & $\begin{array}{c}\text { Média de grupo } \\
\text { controle }\end{array}$ & Média SD - estudo \\
\hline Linguagem & 70,8 & 103,7 & 83,07 \\
\hline Motora & 62,3 & 102,3 & 78,31 \\
\hline
\end{tabular}

\section{DISCUSSÃO}

Os resultados deste estudo, assim como outros já realizados, apontam que o desenvolvimento psicomotor em crianças com SD ocorre, em maior ou menor grau, com atraso em todos os domínios avaliados, quando comparado ao de crianças com desenvolvimento típico. Ainda, esta pesquisa buscou verificar o DPM de crianças com SD, de até 42 meses, considerando os aspectos cognitivo, de linguagem (comunicação receptiva e expressiva) e motor (motricidade fina e grossa) por meio da Escala de 
Desenvolvimento infantil Bayley III em crianças que em algum momento das suas vidas realizaram estimulação precoce, fisioterapia e/ou fonoaudiologia.

No domínio cognitivo, o presente estudo demonstra, a partir da pontuação escalonada, que 12 crianças se apresentaram a 1 DP abaixo da média e uma criança ficou classificada entre 1 e 2 DP abaixo da média. Esses dados são positivos, tendo em vista que nenhuma das crianças foi classificada em um nível mais baixo de desenvolvimento cognitivo. Setenta crianças com o diagnóstico de SD, divididas em 3 grupos: 1) crianças $<3$ anos, 2) crianças entre 3 e 6 anos e, 3) crianças $>6$ anos, foram avaliadas com os protocolos da Medida da Função Motora Grossa-88 (GMFM88), Escala de Equilíbrio Pediátrico (PBS), Escala BrunetLezine (para menores de 3 anos) e a Escala de Inteligência Wechsler - WISC (para maiores de 3 anos) e mostraram que $70 \%$ das crianças foram classificadas com grau de comprometimento moderado nos quesitos desenvolvimento mental e psicomotor; apenas $4 \%$ foram identificadas como tendo comprometimento grave $e$ as demais foram caracterizadas como normal ou leve ${ }^{8}$. Apesar de o grupo avaliado ser composto por crianças um pouco mais velhas do que os participantes de nosso estudo, os resultados confirmam os aqui encontrados, visto que todas as crianças avaliadas neste estudo foram classificadas com desempenho cognitivo médio, média baixa e limítrofe; nenhuma foi classificada como grave. 
Cinquenta e um indivíduos com SD, com idades entre 6 e 24 anos, foram avaliados com o teste não-verbal de inteligência - TONI 3. Os resultados apontaram que 72,6\% da amostra obteve pontuação baixa, caracterizando comprometimento cognitivo ${ }^{9}$. Os mesmos resultados foram observados em 60 indivíduos com SD, com idades entre 5 e $17 \operatorname{anos}^{10}$. Desses, 41 foram testados com a Escala de Inteligência Wechsler Pré-Escolar e Primária $3^{a}$ Edição (WPPSI-III), 7 com a escala de inteligência Wechsler, $4^{a}$ edição e uma com a Leiter International, Escala de Desempenho - Revisada. No grupo de 37 crianças de 5 a 12 anos, 35\% tinham deficiência intelectual (DI) leve e 65\% foram considerados DI graves. O autor salienta que não foi possível realizar testes cognitivos formais em 11 crianças devido à DI muito grave. Na faixa etária de 13 a 18 anos, num total de 23 adolescentes com SD, 9\% foram classificados com DI leve e $91 \%$ com DI grave, ou seja, os mais velhos, na faixa etária de 13 a 18 anos, apresentaram uma porcentagem maior de DI grave, se comparado às crianças na faixa etária de 5 a 12 anos. Em parte, esses achados reforçam o encontrado em nosso estudo, no qual as crianças obtiveram um desempenho positivo na avaliação cognitiva, não havendo nenhuma criança com grau grave, visto que as pontuações variaram entre média, média baixa e limítrofe, conforme pressupostas da Bayley III.

Com relação à área de linguagem, em nosso estudo, ao observar-se, separadamente a comunicação expressiva e receptiva, pode-se verificar, pela pontuação escalonada, que 
a área de comunicação expressiva possui pontuação mais baixa do que a área de comunicação receptiva. Em estudo com 30 crianças (10 com SD e 20 com desenvolvimento típico), a média de idade cronológica (IC) foi de 39,46 meses, por meio do instrumento "Perfil Psicoeducacional Revisado" PEP- $R$, que avalia a Idade de Desenvolvimento em 7 áreas: imitação, percepção, motora fina, motora ampla, coordenação, viso-motora, desempenho cognitivo (compreensão de linguagem) e cognição verbal (expressão de linguagem $)^{11}$. O estudo observou que enquanto a compreensão da linguagem apresenta-se de semelhante à IC, a linguagem expressiva verbal apresenta atraso significativo ${ }^{11}$. A média de desenvolvimento da área de linguagem expressiva, nesse perfil de crianças, indica níveis abaixo da metade da idade média de desenvolvimento, apontando que os déficits de linguagem expressiva são significativamente maiores do que $o$ atraso global da criança com SD, reforçando os nossos achados.

Por meio da classificação descritiva também é possível observar que 6 crianças estão no limite do que se espera para suas faixas etárias; 6 apresentam média baixa e uma está na média, segundo critérios da Bayley III. Estudo realizado com 28 crianças com SD, atendidas no Laboratório de Investigação Fonoaudiológica do Curso de Fonoaudiologia da Faculdade de Medicina da Universidade São Paulo, com o objetivo de verificar o desempenho de crianças com SD no que diz respeito ao modo comunicativo (verbal, vocal e gestual) verificou que o G1, composto por 9 crianças com SD 
com idades entre 2 e 3 anos e meio, utilizou significativamente mais gestos (76\%) quando comparadas às crianças mais velhas ${ }^{12}$. Crianças com SD, para compensar o atraso na produção oral e a ininteligibilidade de fala, passam a desenvolver de modo significativo a comunicação gestual, pois quando elas não são compreendidas, os sinais gestuais constituem um meio mais confiável do que a fala, podendo residir nesse fato 0 baixo desempenho na comunicação verbal, conforme resultados do presente estudo ${ }^{13,14}$.

Neste trabalho, no qual foram avaliadas apenas duas crianças mais velhas (29 meses e 40 meses), observou-se que elas se apresentam no limite de desenvolvimento na área de linguagem, enquanto as mais novas (de 10 e 12 meses) estão na média baixa ou média, conforme proposto na Bayley III. O mesmo aconteceu no estudo realizado com 18 crianças com SD e média de idade de $6 \pm 2,04$ anos, por meio da Escala de Desenvolvimento Motor (EDM) ${ }^{15}$. Ao avaliarem os domínios de linguagem e organização temporal, os autores verificaram que quanto maior a idade da criança, mais déficits ela apresenta nessas áreas. Em outro estudo que avaliou 10 crianças com SD, com idades entre 1 e 5 anos, por meio de um roteiro de observação do comportamento motor para descrever os padrões de locomoção, de manipulação, de estabilidade e de percepção e provas piagetianas adaptadas para a descrição da construção das noções de objeto permanente, de causalidade física, de espaço e de tempo concernentes ao 
estágio sensório-motor do desenvolvimento cognitivo e de linguagem, observou-se que os principais déficits motores e as maiores defasagens cognitivas e de linguagem foram apresentadas pelas crianças mais novas da amostra ${ }^{16}$. Da mesma forma, uma maior interdependência na relação entre os diferentes itens avaliados ocorreu nesses mesmos participantes. Os autores acreditam, que com o passar da idade, o processo de desenvolvimento dessas crianças tenda a se aproximar do que é tipicamente esperado, ratificando a importância das intervenções e estimulações, em longo prazo, para crianças com SD.

Em estudo com crianças com SD que possuíam a trissomia 21, a hipotonia não está presente apenas nos músculos maiores do corpo, aparece também nos músculos faciais e orais, alterando a arcada dentária e o vedamento labial, fazendo com que a língua fique projetada para fora da boca ${ }^{17}$. Há, na maioria dos casos, hipodesenvolvimento do palato e da maxila, reduzindo o tamanho da cavidade oral; essas alterações acarretam uma maior dificuldade de deglutição, mastigação e linguagem justificando os atrasos. Para os autores, os resultados de baixo desempenho na avaliação da linguagem expressiva verbal podem ser associados à hipotonia, alterações de palato e da língua.

Ao analisar nossos resultados, considerando as pontuações escalonada, composta, equivalente de idade e classificação descritiva, é possível verificar que as áreas de linguagem expressiva e de motricidade grossa são as mais defasadas, tendo essa última a menor pontuação dentre os 
domínios testados, visto que nenhuma das crianças ficou na média de desenvolvimento, estando sempre abaixo do esperado.

Ao avaliar 19 crianças com SD e 25 crianças com desenvolvimento típico, observou que crianças com SD precisam de mais tempo para atingir os marcos motores, pois ficam mais tempo aprimorando as habilidades ${ }^{18}$. As maiores dificuldades observadas nesse estudo estão relacionadas à aquisição das posições prona, sentada e em pé, pois cada vez mais as crianças, nesses estágios, são desafiadas pela gravidade.

Ao observar os resultados da Tabela 1 , de equivalente de idade, é possível notar que quanto mais a criança cresce, mais ela demora para atingir os marcos motores; a criança mais nova, com 10 meses, apresenta motricidade grossa compatível com a de uma criança de desenvolvimento típico de 8 meses e a mais velha, de 40 meses, apresenta motricidade grossa semelhante ao que se espera para criança de 20 meses.

A criança com SD atinge os principais marcos motores mais tarde, sendo o sentar em torno de 9 meses, ficar em pé com apoio com 15 meses, e andar por volta dos dezenove meses com uma característica forte, de base alargada, que se dá por alterações esqueléticas como excessiva rotação externa e abdução de quadril ${ }^{19}$. O atraso na aquisição dessas habilidades irá interferir em outras áreas.

Também foi observado atraso no desenvolvimento motor em 80 crianças com SD frequentadoras da APAE de 
Salvador ${ }^{20} ; 85 \%$ obtiveram controle cervical com mais de 4 meses; o sentar foi alcançado após os 8 meses de idade em $58 \%$ dos indivíduos; $57 \%$ não engatinhavam e, os que engatinhavam com mais de 1 ano somaram $31 \%$. O valor mais significativo encontrado foi quanto à deambulação, pois $81 \%$ das crianças com SD andaram somente após os 16 meses. Apesar do ritmo de aquisição das habilidades psicomotoras ser lento, é um ritmo crescente de evolução ${ }^{18}$. A criança com SD seguirá a mesma sequência de desenvolvimento do que a criança com desenvolvimento típico, entretanto, necessitará de mais tempo para adquirilas.

Em relação à avaliação da motricidade fina das crianças de nossa amostra, por meio da pontuação escalonada, percebe-se que todas as crianças atingiram pontuações entre 10 e 7. Ao avaliar o desenvolvimento de 12 crianças com SD e de 12 crianças com desenvolvimento típico, por meio da escala Bayley III e do subteste de motricidade fina, em comparação com crianças com desenvolvimento típico, as crianças com SD possuem maior atraso no desenvolvimento na motricidade fina ${ }^{21}$. Os achados dos autores indicam que a maioria das crianças com SD foi classificada com atraso leve nessa área, tendo em vista que dois participantes obtiveram pontuação entre 10 e 7 e 9 entre 7 e 4 e uma obteve pontuação 3 .

No intuito de possibilitar o uso da Bayley III na avaliação de crianças com desenvolvimento "atípico", para sua validação, foram avaliadas 90 crianças com SD, crianças 
com atraso na linguagem secundário à SD, nascidas prematuramente ou pequenas para a idade gestacional, ou ainda, em risco de atraso psicomotor devido a outros fatores. Nenhuma das crianças que possuía medição de QI (quociente de inteligência) superior a 75 ou inferior a 55 foi qualificada para o estudo7. Nesse caso, as pontuações obtidas foram menores para todas as áreas, quando comparadas ao desenvolvimento de crianças com desenvolvimento típico ${ }^{7}$. Se compararmos as pontuações obtidas pelas crianças do presente estudo com as pontuações obtidas pelas crianças com SD avaliadas durante a validação do protocolo da Bayley III, pode-se verificar que apenas a área de motricidade grossa em nosso estudo ficou mais próximo dos resultados do protocolo, cuja média foi de 3,4 e em nosso estudo 4,61. As demais áreas avaliadas ficaram com uma média melhor do que as crianças com SD que participaram do protocolo, sendo que nossas médias se aproximam mais da média de crianças com desenvolvimento típico, do que das crianças com SD avaliadas durante a validação do protocolo da Bayley III. A explicação pode residir no fato de nosso $n$ (13) ser bem menor do que o $n$ (90) testado e avaliado pelos administradores do protocolo Bayley III. Ressalta-se que o QI dos participantes do presente estudo não foi testado; no estudo viabilizado pelos administradores do protocolo da Bayley III, o QI dos participantes foi considerado abaixo da média, baixo ou muito baixo.

Destaca-se, ainda, que não se tem conhecimento do tipo de acompanhamento dos participantes avaliados pelo 
protocolo, e sua validação; as crianças avaliadas em nosso estudo recebem acompanhamento desde o nascimento, com estimulador precoce, fisioterapeuta, fonoaudiólogo, dentre outros, o que poderia justificar a diferença encontrada no comparativo entre as crianças com SD do protocolo e as de nossa amostra.

No contexto deste estudo, vale sublinhar que, independente dos resultados encontradas, mediante utilização de vários instrumentos, protocolos e escalas, em diferentes pesquisas, no que tange ao desenvolvimento psicomotor de crianças com SD, existe um consenso atual da sociedade científica de que não há graus da SD e que as diferenças de desenvolvimento procedem das características pessoais decorrentes de herança genética, estimulação, educação, meio ambiente e problemas clínicos, entre outros 22 .

\section{CONCLUSÃO}

Dado o tamanho de nossa amostra, os dados aqui apresentados não podem ser generalizados, entretanto, foi possível verificar que as crianças com Síndrome de Down possuem atraso em todas as áreas avaliadas: cognitiva, comunicação receptiva e expressiva, motricidade fina e grossa, quando comparadas com crianças com desenvolvimento típico. Assim como citado por outros autores, as maiores defasagens estão relacionadas à linguagem expressiva e motricidade grossa. Sugere-se a 
realização de novos estudos, com a participação de um maior número de crianças, para que os resultados sejam ratificados.

\section{REFERÊNCIAS}

1.Langdon J, Down JLH. Observations on an Ethnic Classification of Idiots. Heredity 1966;21:695-97.

https://doi.org/10.1038/hdy.1966.69

2.Godzicki B, Silva PA da, Blume LB. Aquisição do sentar independente na Síndrome de Down utilizando o balanço. Fisioter Mov 2010;23:7381. https://doi.org/10.1590/S0103-51502010000100007

3. Diretrizes de atenção à pessoa com Síndrome de Down (endereço na internet). Brasília: Ministério da Saúde, 2012 (acessado em: 04/2019). Disponível em:

http://bvsms.saude.gov.br/bvs/publicacoes/diretrizes cuidados sindr ome down.pdf

4.Sampaio AM. A Síndrome de Down no Contexto Familiar e Social. Rev Even Pedagog 2012;3:276-86. https://doi.org/10.30681/22363165

5.Lira BS. A Importância da Psicomotricidade para o Desenvolvimento de Crianças com Síndrome de Down (Trabalho de Conclusão de Curso). Campina Grande: Universidade Federal de Campina Grande, 2015.

6.Rodrigues OMPR. Escalas de desenvolvimento infantil e o uso com bebês. Educar Rev 2012;43:81-100.

https://doi.org/10.1590/S0104-40602012000100007

7.Bayley N. Bayley: Escala de desenvolvimento do bebê e da criança pequena. 3. ed. São Paulo: Editora Pearson Clinical Brasil, 2018.

8. Malak R, Kotwicka M, Krawczyk-Wasielewska A, Mojs E, Samborski W. Motor skills, cognitive development and balance functions of children with Down syndrome. Ann Agricul Environ Med 2013;20:8036. http://www.aaem.pl/Motor-skills-cognitive-development-andbalance-functions-of-children-with-Down-syndrome, $72020,0,2 . \mathrm{html}$

9.Pacanaro SV, Santos AAA, Suehiro ACB. Avaliação das habilidades cognitiva e viso-motora em pessoas com Síndrome de Down. Rev Bras Educ Esp 2008;14:311-26.

https://doi.org/10.1590/S1413-65382008000200011

10.Oxelgren UW, Myrelid A, Annerén G, Westerlund J, Gustafsson J, Fernell $\mathrm{E}$. More severe intellectual disability was found in teenagers than younger children with Down syndrome. Acta Pæd 2018;108:9616. https://doi.org/10.1111/apa.14624

11. Brandão SRS. Desempenho na linguagem receptiva e expressiva de crianças com Síndrome de Down (Tese). Santa Maria: Universidade Federal de Santa Maria; 2006. 
https://repositorio.ufsm.br/bitstream/handle/1/6532/Dissertacao\%20 Silvia\%20Regina\%20Brandao.pdf? sequence $=1$

12.Porto-Cunha E, Limongi SCO. Modo comunicativo utilizado por crianças com síndrome de Down. Pró-Fono Rev Atual Cient 2008;20:243-8.

https://doi.org/10.1590/S0104-56872008000400007

13. Franco F, Wishart JG. Use of pointing and other gestures by young children with Down syndrome. Am J Mental Retard 1995;100:160-82. https://www.researchgate.net/publication/14673603_Use_of_pointin g_and_other_gestures_by_young_children_with_Down_syndrome_A merican_Journal_of_Mental_Reta rdation_100_160-182

14.Chan JB, Iacono T. Gesture and production in children with Down syndrome. Augmentative and Alternative Communication 2001;17:7387. https://doi.org/10.1080/714043370

15. Oliveira MCS, Silva FA, Bofi TC, Carvalho AC. O Desempenho da Linguagem e Organização Espaço-temporal em Crianças com Síndrome de Down por Meio da Escala de Desenvolvimento Motor. Colloq Vitae 2014;6:94-101.

http://revistas. unoeste. br/revistas/ojs/index.php/cv/article/viewFile/ $1200 / 1265$

16. Bonomo LMM, Rossetti CB. Aspectos percepto-motores e cognitivos do desenvolvimento de crianças com Síndrome de Down. Rev Bras Cresc Desenv Hum 2010;20:723-34.

http://pepsic.bvsalud.org/pdf/rbcdh/v20n3/07.pdf

17. Macho VMP, Seabra M, Pinto A, Soares D, Andrade C. Alterações craniofaciais e particularidades orais na trissomia 21. Acta Ped Port 2008;39:190-4. https://doi.org/10.25754/pjp.2008.4600

18.Pereira K. Perfil do Desenvolvimento Motor de Lactentes com Síndrome de Down dos 3 aos 12 Meses de Idade (Tese). São Carlos: Universidade Federal de São Carlos, 2008. https://repositorio.ufscar.br/handle/ufscar/5092?show=full.

19.Schwartzman JS. Síndrome de Down. 2o ed. São Paulo: Memnon edições científicas; 2003.

20. Matos MA. Instabilidade atlantoaxial e hiperfrouxidão ligamentar na síndrome de down. Acta Ortop Bras 2005;13:165-7.

http://dx.doi.org/10.1590/S1413-78522005000400001

21. Coopede AC, Campos AC, Santos DCC, Rocha NACF. Desempenho motor fino e funcionalidade em crianças com síndrome de Down. Fisioter Pesq 2012;19:363-8.

http://dx.doi.org/10.1590/S1809-29502012000400012

22.Silva MFMC, Klheinhans ACS. Processos cognitivos e plasticidade cerebral na Síndrome de Down. Rev Bras Ed Esp 2006;12:123-38. http://dx.doi.org/10.1590/S1413-65382006000100009 